\title{
Chapter 9 \\ The Development of Incremental Sheet Forming from Flexible Forming to Fully Integrated Production of Sheet Metal Parts
}

\author{
Gerhard Hirt, Markus Bambach, Wolfgang Bleck, Ulrich Prahl \\ and Jochen Stollenwerk
}

\begin{abstract}
Incremental Sheet Forming (ISF) was devised as a flexible forming process in the 1990s. The basic principle of ISF is that a generic forming tool moves along a tool path and progressively forms a metal sheet into the desired shape. The tool is either moved using $\mathrm{CNC}$ machines or industrial robots. Applying CNC technology or robots to sheet metal forming allows for replacing expensive dedicated tooling and for a fast transfer from the CAD model to the formed part. Since its first applications in the 1990s ISF has undergone tremendous developments. Various process variants such as double-sided ISF and hybrid process combinations such as heat-assisted ISF as well as stretch-forming and ISF have been put forward. The present contribution gives an overview of these developments with a special focus on the outcome of the research accomplished within the cluster of excellence "Integrative Production Technology for High Wage Countries", where the development of fully integrated sheet metal production facilities is envisioned as the next evolution step of ISF. The development of dedicated equipment for hybrid and fully integrated sheet metal manufacturing and specialized CAX environments as well as applications are described to show the potential of the technology.
\end{abstract}

G. Hirt $(\square) \cdot$ M. Bambach

Metal Forming Institute (IBF), RWTH Aachen University,

Intzestr. 10, 52056 Aachen, Germany

e-mail: hirt@ibf.rwth-aachen.de

W. Bleck · U. Prahl

Department of Ferrous Metallurgy (IEHK), RWTH Aachen University,

Intzestr. 1, 52072 Aachen, Germany

e-mail: bleck@iehk.rwth-aachen.de

J. Stollenwerk

Fraunhofer Institute for Laser Technology ILT, Steinbachstr. 15,

52074 Aachen, Germany

e-mail: jochen.stollenwerk@ilt.fraunhofer.de

(C) The Author(s) 2015

C. Brecher (ed.), Advances in Production Technology,

Lecture Notes in Production Engineering, DOI 10.1007/978-3-319-12304-2_9 


\subsection{Introduction to Incremental Sheet Metal Forming}

Incremental sheet forming (ISF) is a flexible forming process for small batch manufacturing and rapid prototyping of almost arbitrary 3D shapes. In ISF, a clamped sheet metal is progressively formed by a moving forming tool (Fig. 9.1, right). In contrast to conventional sheet metal forming processes such as deep drawing (Fig. 9.1, left), only a single die is needed, which does not have to be a full male or female die but can be a partial support.

The tool path covers the surface of the desired product, similar to the finishing stage in z-level machining. In every instant of the forming process in which the tool moves over the sheet metal, localized plastic deformation is produced and the final part shape is the result of all localized plastic deformation events. Several variants of the incremental sheet forming process have been developed in the past:

- Conventional ISF. Conventional ISF comprises the variants of 'single point incremental forming' (SPIF) and 'two-point incremental forming' (TPIF). In SPIF, either no support at all or only a simple rig is used to support the outer contour of the part. In TPIF, the sheet metal is formed over a full or partial positive die.

- Double-sided ISF. In this process variant, a tool is used on either side of the sheet, with one tool acting as the master forming tool and the other one acting as a local support. This process was investigated by Meier et al. (2007), Maidagan et al. (2007) as well as Malhotra et al. (2011).

- Stretch-forming and ISF. To overcome some of the limitations of conventional ISF process variants such as the long process time, the pronounced thinning and
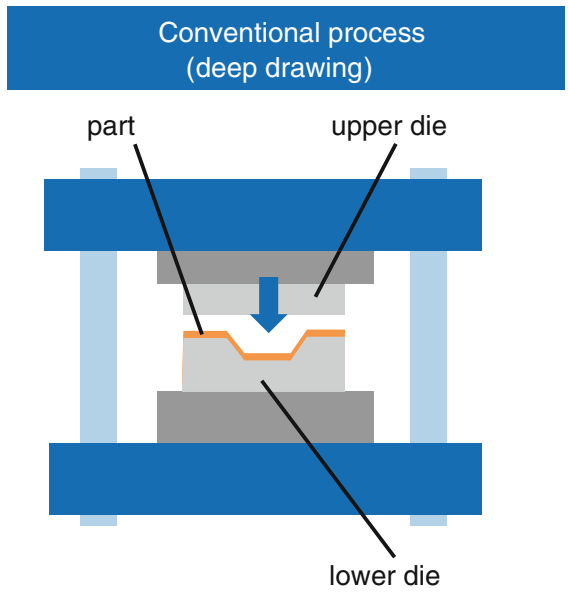
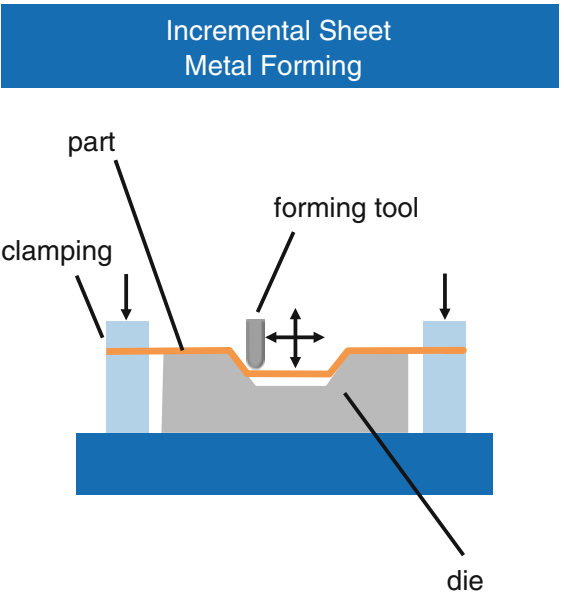

Fig. 9.1 Illustration of conventional deep drawing and 'single point incremental forming' (SPIF) 
the limited geometrical accuracy, ISF was combined with stretch-forming to a hybrid process by Taleb Araghi et al. (2009). The process was performed on a dedicated machine that combines four stretch-forming units and a CNC unit for ISF.

- Heat-assisted ISF. In order to form e.g. titanium or magnesium alloys with a low formability at room temperature, heat-assisted ISF variants were developed, such as laser-assisted ISF by Duflou et al. (2007) and Göttmann et al. (2011), or ISF with resistance heating, see e.g. Göttmann et al. (2012). These variants are also hybrid processes, whose set-up and control is much more involved than for conventional ISF.

Both conventional ISF and the newer process variants have been developed with great effort by a number of research groups, but up to now only with limited industrial take-up. The main limitations of conventional ISF are (i) the limited geometrical accuracy, (ii) excessive sheet thinning, (iii) the long process time and (iv) the need for dedicated CAE tools. Besides that, potential markets for ISF are sheet metal parts made from titanium and magnesium alloys which are hard to form at room temperature and require forming at elevated temperature.

To meet the above-mentioned challenges and to make ISF viable in an industrial context, various technological developments beyond conventional ISF are necessary. This contribution gives an overview of recent developments in ISF with a strong focus on hybrid ISF processes developed in the cluster of excellence "integrative production technology for high-wage countries".

The paper is organized as follows: The next section gives an overview of the design of the dedicated machine for hybrid ISF processes and the CAX tools needed to operate the machine. The capabilities of the hybrid processes of stretch forming and ISF as well as laser-assisted ISF are demonstrated using case studies. Finally, the benefit of the hybrid processes compared to standard ISF is summarized.

\subsection{Design of a Machine for Hybrid ISF}

\subsubsection{Basic Set-up for Stretch-Forming and ISF}

The machine shown in Fig. 9.2 is based on a standard milling machining center into which four dedicated stretch-forming modules were integrated (shown in blue in Fig. 9.2). Thus, the process steps of milling of the die, stretch forming, ISF and trimming can be carried out on a single machine.

Particularly noteworthy is the stiff machine bed (Fig. 9.2, right), which must bear the extremely high process forces exerted by stretch-forming (about $200 \mathrm{kN}$ per element) in a confined space. Furthermore, an interface has been created in the 5-axis head which can receive forming tools for ISF as well as conventional milling tools. For ISF a force limiter was developed to protect the linear and rotary axes of the milling machine from overloading. Both for stretch forming as well as for ISF a 

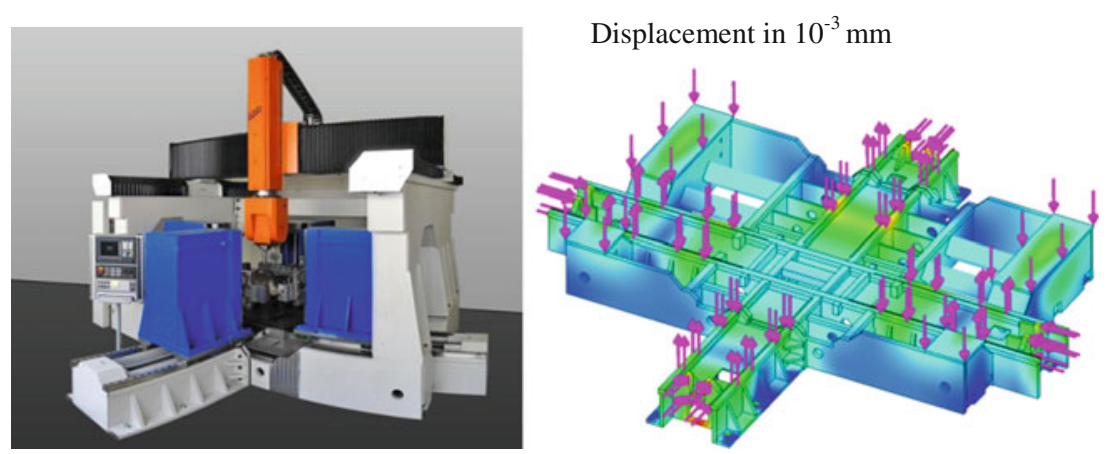

Fig. 9.2 (Left) Hybrid machine enabling stretch forming and ISF. (Right) simulation of the deflection of the machine bed

workpiece holder is required, which must transmit the high process forces. The stretch-forming modules allow movements in horizontal and vertical direction and have a hinged clamp. This is necessary to allow for tangential stretch forming. The clamping of the workpieces is self-adjusting and is designed for sheet metal of 1-4 mm thickness. All movements are performed by NC controlled linear axes.

During milling of the die needed for stretch forming, the machine can be used as 5-axis milling machine with three linear axes and two rotary axes. With the system, the commonly used mold materials for ISF (aluminum, plastic, wood) can be machined.

The machine is equipped with a CNC control Siemens 840 D NCU 573 SL. Due to the flexible architecture of the controller it is possible to integrate special control functions for the stretch forming and incremental sheet forming directly in the controller. This functionality has been used to integrate a laser system as further axis (see below). The technical data of the installed system are summarized in Table 9.1.

Table 9.1 Technical data of the hybrid machine for stretch forming and ISF

\begin{tabular}{|c|c|c|c|c|c|}
\hline Control & \multicolumn{5}{|c|}{ Siemens 840 D NCU 573 SL } \\
\hline \multirow[t]{2}{*}{ Accuracy } & \multicolumn{3}{|c|}{ Positioning accuracy } & \multicolumn{2}{|c|}{ Repeat accuracy } \\
\hline & \multicolumn{3}{|c|}{ $\pm 0.03 \mathrm{~mm}$} & \multicolumn{2}{|c|}{ $\pm 0.015 \mathrm{~mm}$} \\
\hline Machine axes & $\mathrm{X}$ & $\mathrm{Y}$ & $\mathrm{Z}$ & SZ horizontal & SZ vertical \\
\hline Traversing range $[\mathrm{mm}]$ & 2.800 & 2.300 & 1.000 & & \\
\hline Teed rate $[\mathrm{m} / \mathrm{min}]$ & 40 & 40 & 20 & & \\
\hline Forming force $[\mathrm{kN}]$ & 4 & 4 & 4 & 200 & 100 \\
\hline \multirow[t]{2}{*}{ Spindle } & Power & \multicolumn{2}{|c|}{ Rotation speed } & Torque & Tool holder \\
\hline & $24 \mathrm{~kW}$ & \multicolumn{2}{|c|}{$18.000 \mathrm{U} / \mathrm{min}$} & $38 \mathrm{Nm}$ & HSK $63 \mathrm{~A}$ \\
\hline
\end{tabular}




\subsubsection{Basic Set-up for Laser-Assisted ISF}

To allow for localized heating, a laser optic was designed and integrated into the machine. The selected laser was a "LDF 10000" diode laser from the company Laserline. The maximum available output of $10 \mathrm{~kW}$ (radiation power) is sufficient to heat common sheet forming materials up to temperatures above $1000{ }^{\circ} \mathrm{C}$. The main advantages of a diode laser are that the beam can be guided via an optical fiber. Thus, the energy required for heating can be directed right to the forming area. The movements of the forming tool can be compensated for by the optical fiber and a feed device.

Since the optical system cannot be rotated around the tool, it was designed so that the laser beam is rotated to the desired position around the tool axis. Rotation of mirrors in the laser optics causes the laser beam to move on a circle. The shape and position of the laser spot can be influenced by selecting different lenses and varying the distances between the mirror components. In the simplest version, a circular laser spot with a diameter of $35 \mathrm{~mm}$ is projected onto the surface of the part at a distance of $45 \mathrm{~mm}$ from the tool axis. The optical system described is fixed to the forming head of the hybrid machine (Fig. 9.3). The beam source used is outside the machine, so that the laser beam has to be guided to the processing point via a fiber optic. The optical system moves together with the processing head during the forming process. The laser spot is positioned by a motor that is built into the optical system.

\subsubsection{CAX Environment}

The CAX environment must provide suitable software tools to plan each step of the combined stretch-forming and ISF process chain as well as for laser-assisted ISF.

Fig. 9.3 Hybrid forming machine with built-in optical system

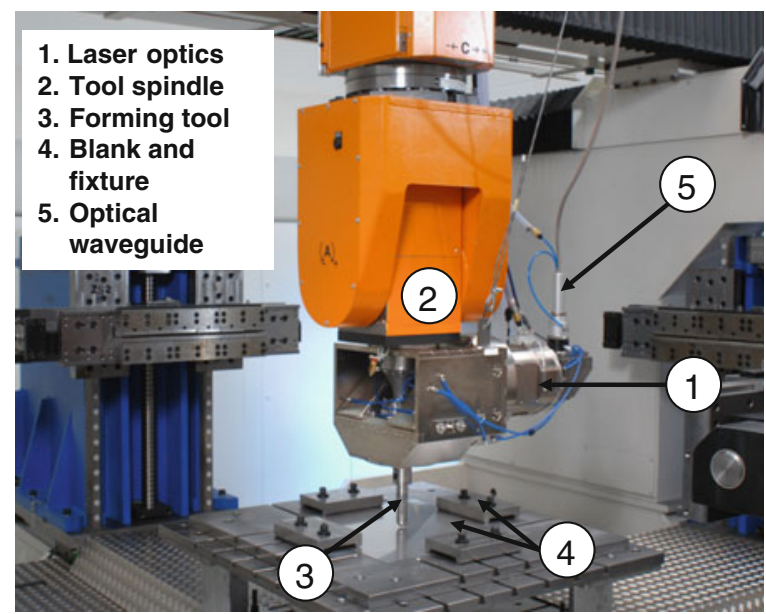


Due to the complexity of the tool kinematics of ISF purely manual machine operation would not be possible. The same holds for stretch forming with up to 8 axes. Due to the novelty of the combination of stretch-forming and ISF process, the development of new CAM features was necessary which do not exist as standard features in common CAX systems.

The programming of the stretch-forming operation is supported by the CAM system, but it is also possible to operate the stretch-forming modules manually and to read back the trajectories into the CAM system. Previously used ISF strategies can be implemented, customized and extended. The simulation and collision checking of the forming tool, the stretch motion of the machine and the fixture situation were another important requirement.

The development of a completely new and independent CAX solution would have cost a tremendous effort. For this reason, the development of the CAX solution was carried out based on the standardized CAX platform NX from Siemens. A key criterion for the selection of NX as CAD/CAM platform is the ability to integrate own functions in the system via programming interfaces and thus to implement specific functions for stretch forming and ISF. NX offers several programming interfaces (APIs) such as NXopen (C, C++, Visual Basic).

The CAM module in NX provides basic functions for milling which can be adapted for ISF. The most important function is the "Z-level" processing. This machining strategy can be programmed with NX both in a 3-axis and with simultaneous 5-axis motion. All process steps for the production of demonstration components-geometry processing, stretch-forming, ISF and trimming the component - can be performed consistently with the developed CAX-chain.

Building on the experience gained during initial manual programming of the stretch forming units, it was possible to automate some repetitive steps. Prestretching of the sheet, approaching the die and bending can be combined within a single smooth trajectory. Since the stretch-forming modules move in planes, the motion can be prescribed by curves in $2 \mathrm{~d}$ space. These can be defined separately for each stretch forming module. For the individual forming steps, the relevant parameters in the form of input values can be defined (Fig. 9.4).
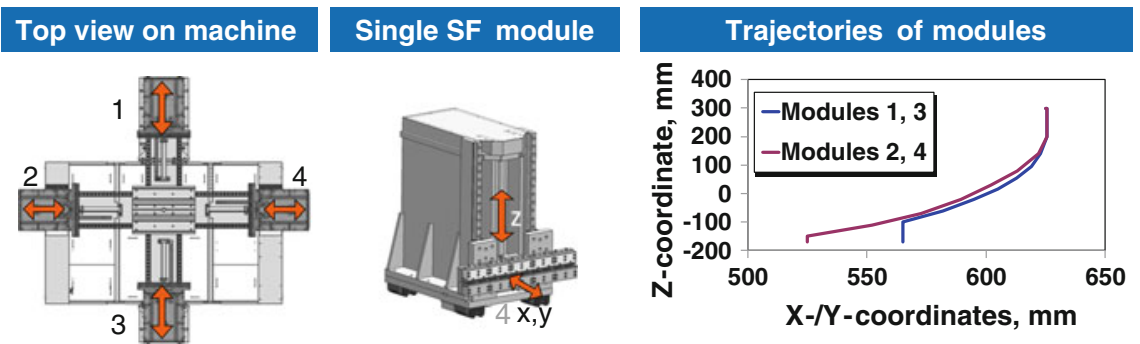

Fig. 9.4 Programming the stretch drawing (left) and graphical representation of the machine kinematics $(r i g h t)$ 


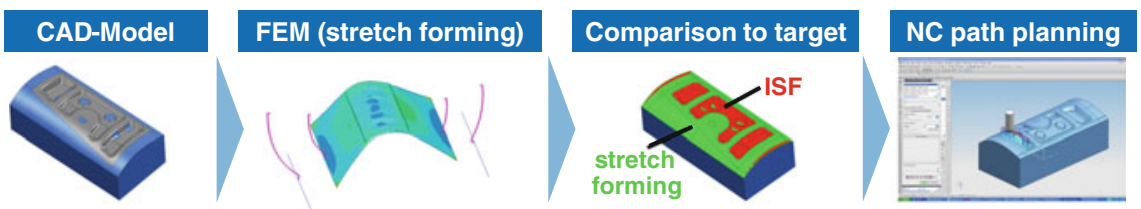

Fig. 9.5 CAD/CAM chain for hybrid stretch forming and ISF

Rotation of laser spot around forming tool

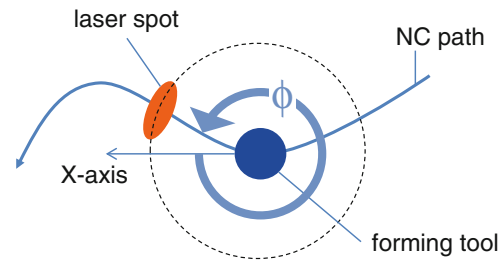

Integration of the laser as additional axis

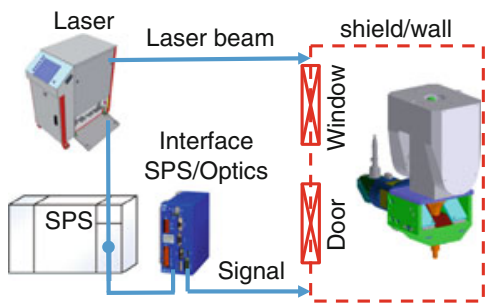

Fig. 9.6 Integration of laser as rotation axis (left) and integration into the machine via SPS (right)

Since stretch forming does not create the final geometry for most parts, the areas that still have to be formed by ISF after stretch forming have to be detected. This is accomplished by reading in results from a finite element simulation of the stretch forming process into the CAD/CAM system. The areas to be formed out by ISF are detected, and tool path planning is done only for the areas shown in red in Fig. 9.5.

The programming of the NC machine tools is often supported by a machine simulation. In the case of the hybrid process, further eight axes for the stretchforming units are present in addition to the five axes of the forming/cutting tool. This underlines the need for system simulation in order to guarantee safe operation. The simulation avoids test runs on the system and therefore contributes significantly to shortening the process planning.

Dedicated CAM tools are also needed for laser-assisted ISF. Special laser optics were devised which guide the laser beam onto a position on the blank that is defined by a rotation angle about the X-axis (Fig. 9.6). The rotation angle is calculated in the CAM system and transferred to the forming machine along with the positioning signals for the forming tool.

\subsection{Case Study: Stretch Forming and ISF}

As an application part, a stiffening frame for a hydraulic access door of an AIRBUS A320 aircraft made of $1.0 \mathrm{~mm}$ stainless steel 1.4541 has been chosen (Fig. 9.7). The part is located on top of the pylon and allows an easy and fast maintenance operation on the hydraulic systems in this area. 

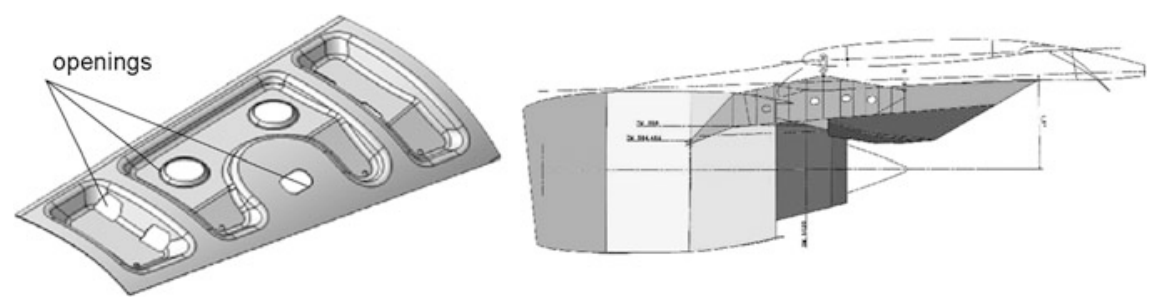

Fig. 9.7 (Left) CAD Drawing of the part. (Right) Position of the part in the airplane

Usually, the part is produced using by a process chain that consists of separate manufacturing operations, i.e., forming and trimming. Especially for small batch sizes, the costs for additional equipment and tooling increase the costs per part. In order to develop an efficient 'integrative production' scenario for small batches, the hybrid machine allows to perform all process steps on a single machine in a single set-up.

To analyze the benefit of the process combination, the part is manufactured both using conventional ISF and with the combination of ISF and stretch-forming. Both parts were trimmed to the final geometry using the milling functionality of the machine. In the case of pure ISF, the forming operation was divided into two steps. In the first step, the outer envelope of the part was formed. The pockets were manufactured separately in the second step. The forming with 'SF + ISF' took $60 \mathrm{~min}$. whereas the manufacturing with the conventional ISF process needed $110 \mathrm{~min}$. Using the process combination, the forming time was reduced by about $45 \%$.

Since the shape of the part shows smooth curvature with relatively flat pockets, low strains are expected that should not lead to significant sheet thinning but influence the geometric accuracy. Hence, within this study the dimensional accuracy was investigated. Both parts (pure ISF-part and 'SF + ISF'-part) were digitized using the gom ATOS system. The comparison of the digitized parts to the CAD model yielded the actual geometric deviations.

Figure 9.8 shows the evaluation of the geometric deviation along a longitudinal section. After trimming, pure ISF yields a lower dimensional accuracy compared to the part made by the process combination. In particular, towards the borders of the part, the geometric deviation of the section made by ISF increases strongly. It can be concluded that for the process combination 'SF + ISF', the superimposed tensile stresses due to stretch forming yield a higher dimensional accuracy in the area close to the outer borders of the part and in the transition to the flange region than pure ISF. For stretch-forming, springback compensation procedures through tool modifications could help to increase the dimensional accuracy even more. 


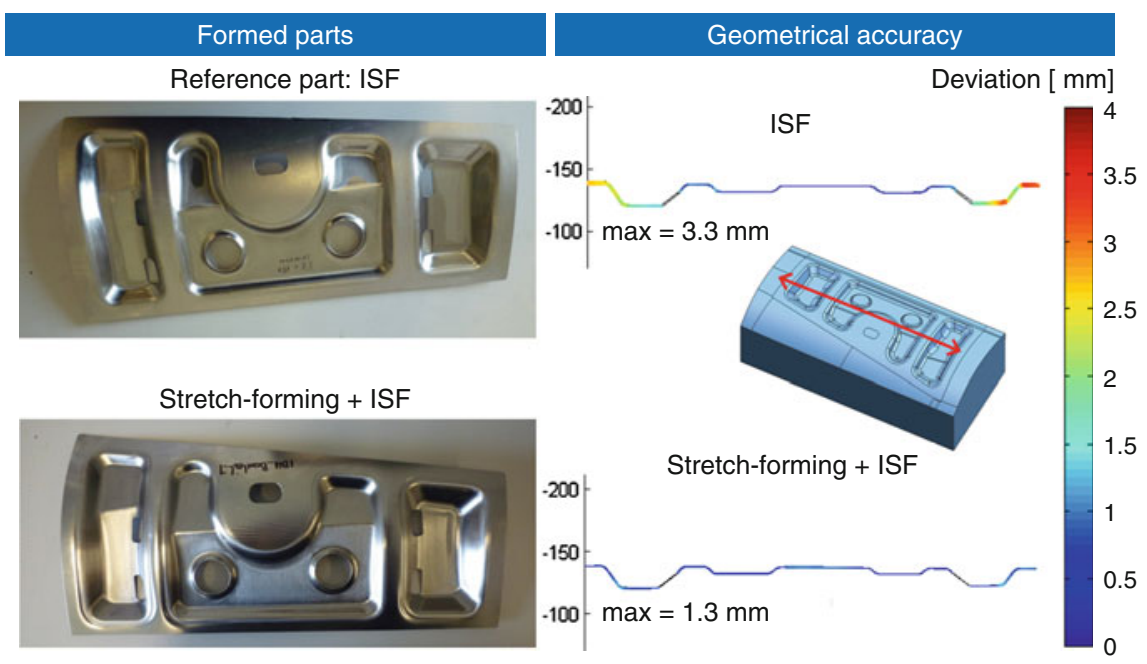

Fig. 9.8 Evaluation of geometric accuracy

\subsection{Case Study: Heat-Assisted ISF}

TiAl6V4 is the most commonly used titanium alloy. At room temperature, formability is very low due to the limited number of glide systems. To analyze the effect of different process parameters such as temperature, strain rate and strain on the deformation behavior of TiAl6V4, processing maps have been developed (Johnson et al. 2003). Vanderhasten et al. (2008) analyzed the deformation behavior of TiAl6V4 by uniaxial tensile testing for a wide range of strain rates and temperatures. However, since ISF is governed by complex stress states in the forming zone, processing maps or uniaxial test data are not representative. In the cluster of excellence, the formability of TiAl6V4 sheets was analyzed by recording forming limit curves, both at room temperature and for slightly elevated temperatures of $300-500{ }^{\circ} \mathrm{C}$. This was accomplished by heating the punch to the respective forming temperature. The FLC in Fig. 9.9 on the left shows that formability increases already in the temperature range of $300-500{ }^{\circ} \mathrm{C}$.

At such low temperatures, oxidation, i.e. the formation of the detrimental $\alpha$-case, does not yet occur during laser ISF since the time spent at that temperature is too short.

Based on the analysis of formability, experiments on $1.5 \mathrm{~mm}$ thick sheet metals of Ti Grade 2 and TiAl6V4 were performed using local laser heating (Fig. 9.9, right). The formed geometry is a cone with a kidney-shaped base with a wall angle of $60^{\circ}$ and a depth of $110 \mathrm{~mm}$ as shown in Fig. 9.8. The pitch between the z-levels was $0.35 \mathrm{~mm}$. The forming velocity was set to $4000 \mathrm{~mm} / \mathrm{min}$. The settings of the laser optics were chosen such that an elliptical laser spot with dimensions $15 \mathrm{~mm} \times 45 \mathrm{~mm}$ was projected onto the sheet metal at a distance of $45 \mathrm{~mm}$ to the tool. The laser power output was controlled using a closed-loop feedback controller. 

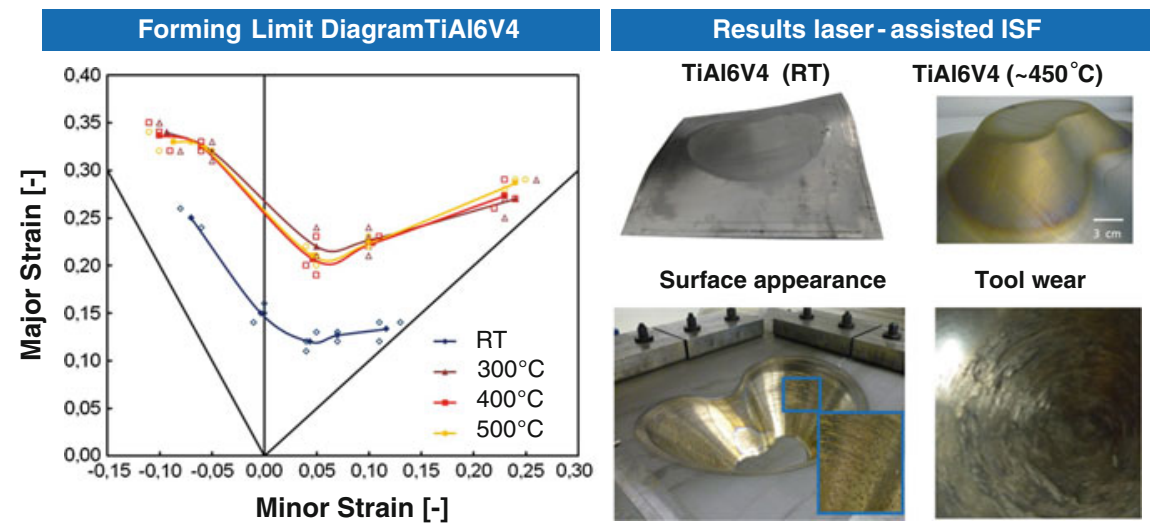

Fig. 9.9 (Left) Formability of TiA16V4 at room temperature and elevated temperature. (Right) Forming of a test shape at room temperature and $\sim 450{ }^{\circ} \mathrm{C}$. Tool wear and surface quality are shown in the bottom

The temperature is measured in the tip of the forming tool $1 \mathrm{~mm}$ below the surface of the forming tool.

Although the part was formed successfully, there is excessive tool wear and the surface quality is poor. Forming of TiAl6V4 using ISF thus requires improved tool concepts such as tools with a rolling instead of a sliding contact.

\subsection{Improvements by the Hybrid ISF Variants}

The most restrictive process limits in conventional ISF are the geometrical accuracy and the strong dependence of sheet thinning on the wall angle of the formed part. Allwood et al. (2005) considered 28 potential sheet metal products from 15 companies to search for potential applications of ISF. A product segmentation approach revealed that only two of the 28 products comply with the capabilities of ISF. In the study, a geometrical inaccuracy of $3 \mathrm{~mm}$ was presumed to exist independent of part size and workpiece material. Although the assumptions made for the achievable tolerance of ISF in the product segmentation approach are oversimplified, they show that the geometrical tolerance is a key factor that decides whether a given product can be manufactured by ISF or not.

Due to the possibility to create a preform by stretch forming and due to the fact that tensile stresses can be superimposed, the combination of stretch forming and ISF helps to improve the geometrical accuracy, as illustrated in Fig. 9.10. In this illustration, it is assumed that the geometrical deviations in ISF scale with the size of the part. 
Fig. 9.10 Improvement of geometrical accuracy by combining ISF with stretch forming (SF)

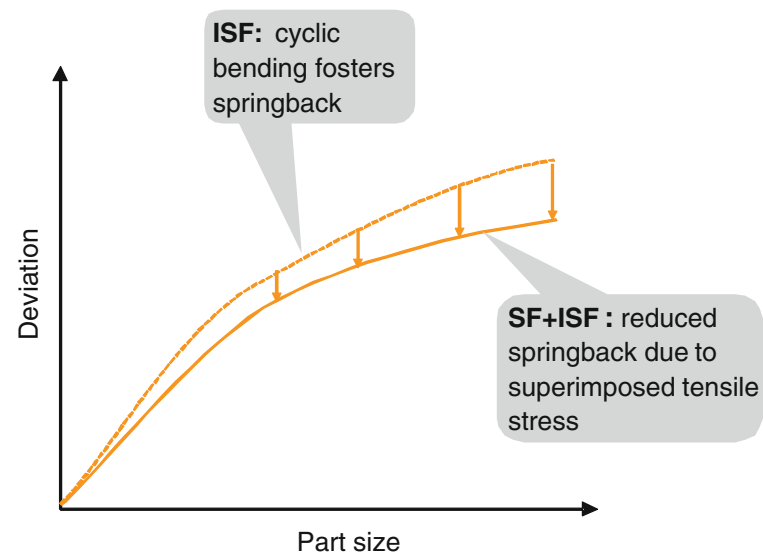

Unlike deep drawing, ISF is a process in which the sheet thickness cannot be held constant. ISF increases the surface area of the part. Thinning in the ISF process is governed by the sine law,

$$
\mathrm{t}_{1}=\mathrm{t}_{0} \sin \left(90^{\circ}-\alpha\right)=\mathrm{t}_{0} \cos (\alpha)
$$

and increases with the wall angle. Thinning in stretch forming does not depend on the wall angle, it is rather governed by frictional constraints. The combination of ISF and SF may hence help improve the process limit determined by excessive thinning. Assuming that the sheet breaks once a certain amount of thinning is reached, forming by stretching should ideally be designed to lead to homogeneous thinning throughout the part so that there is no "weak spot" with maximum thinning. This ideal situation is hard to achieve. However, since thinning in ISF and SF affects different areas of the part, it can be complementary in many cases and hence the thickness reduction can be distributed more evenly over the part, as illustrated in Fig. 9.11. Due to volume constancy, stretching of the sheet must be compensated by thinning, i.e.

$$
S_{0} t_{0}=S_{1} t_{1}
$$

If the surface stretch ratio $\ln \left(S_{0} / S_{1}\right)$ is distributed unevenly over the part, there will be an area of maximum stretching and, correspondingly, maximum thinning. This area is prone to failure. To avoid it, the material should be distributed as homogeneously as possible.

The benefit of forming materials with low formability at room temperature such as titanium and magnesium alloys at elevated temperatures is shown in Fig. 9.12.

Assuming again that a maximum allowable thickness reduction exists, increasing the temperature will increase the limit strain from $\varepsilon_{\max , \mathrm{RT}}$ at room temperature 
Fig. 9.11 Thinning in ISF and the combination of SF and ISF

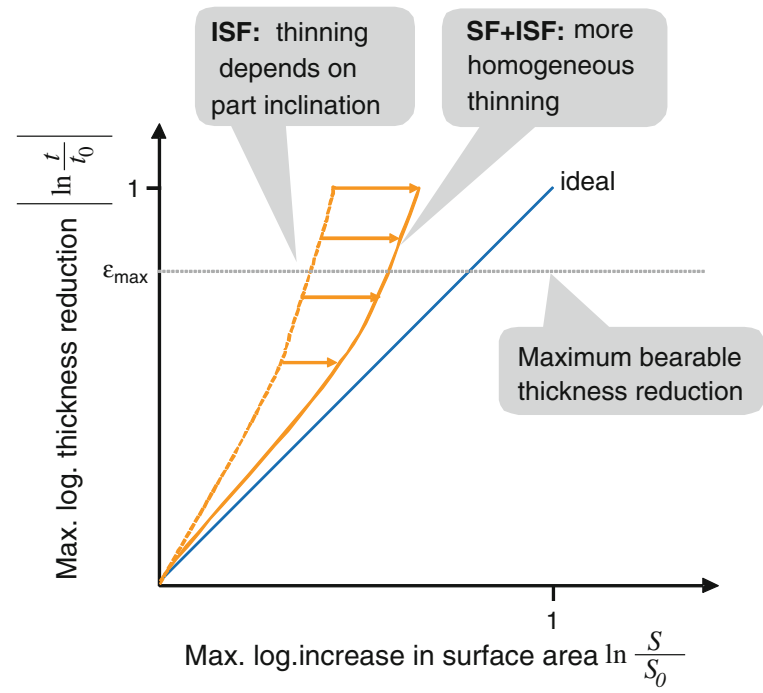

Fig. 9.12 Process limits of ISF at room temperature and elevated temperature

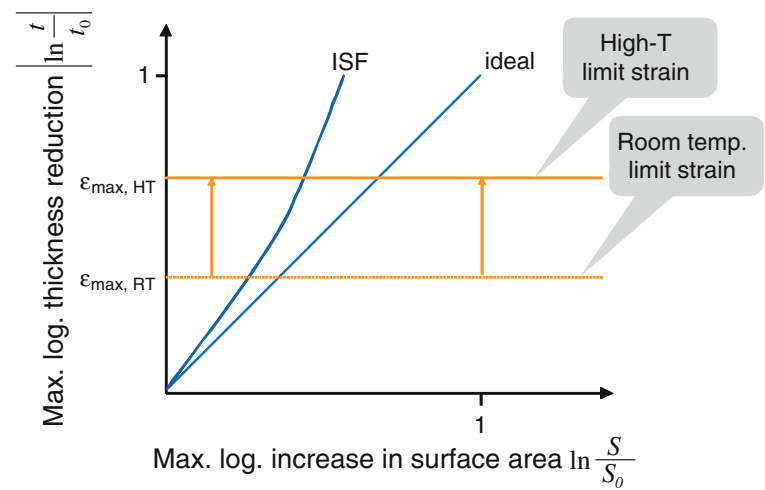

to $\varepsilon_{\max , \mathrm{HT}}$ at high-temperature deformation. This allows a larger increase in surface area and hence forming of more complex parts.

Open Access This chapter is distributed under the terms of the Creative Commons Attribution Noncommercial License, which permits any noncommercial use, distribution, and reproduction in any medium, provided the original author(s) and source are credited.

Acknowledgments The authors would like to thank the German Research Foundation (DFG) for the support of the depicted research within the Cluster of Excellence "Integrative Production Technology for High Wage Countries".

Parts of this research are funded by the German Federal Ministry of Education and Research (BMBF) within the Framework Concept "Research for Tomorrow's Production" (funding number 02PU2104), managed by the Project Management Agency Karlsruhe (PTKA). 


\section{References}

Allwood J, King G, Duflou J (2005) A structured search for applications of the incremental sheetforming process by product segmentation. Proceedings of the I MECH E Part B Journal of Engineering Manufacture 6:239-244

Duflou J, Callebaut B, Verbert J, Baerdemaeker H de (2007) Laser assisted incremental forming: formability and accuracy improvement. CIRP Annals-Manufacturing Technology 56 (1):273-276

Göttmann A, Bailly D, Bergweiler G, Bambach M, Stollenwerk J, Hirt G, Loosen P (2012) A novel approach for temperature control in ISF supported by laser and resistance heating. The International Journal of Advanced Manufacturing Technology:1-11

Göttmann A, Diettrich J, Bergweiler G, Bambach M, Hirt G, Loosen P, Poprawe R (2011) Laserassisted asymmetric incremental sheet forming of titanium sheet metal parts. Production Engineering 5(3):263-271

Johnson AW, Bull CW, Kumar KS, Briant CL (2003) The influence of microstructure and strain rate on the compressive deformation behavior of Ti-6Al-4 V. Metallurgical and Materials Transactions A 34(2):295-306

Maidagan E, . Zettler J, Bambach M, Rodríguez P, Hirt G (2007) A new incremental sheet forming process based on a flexible supporting die system. In: Sheet Metal 2007: Proceedings of the International Conference. Trans Tech Publications Ltd, Uetikon-Zuerich, Switzerland, pp 607-614

Malhotra R, Ren F, Reddy NV, Kiridena V, Cao J, Xia ZC (2011) Improvement of geometric accuracy in incremental forming by using a squeezing toolpath strategy with two forming tools. Journal of Manufacturing Science and Engineering 133(6):61019

Meier H, Smukala V, Dewald O, Zhang J (2007) Two point incremental forming with two moving forming tools. In: SheMet '07, Proceedings of the 12th International Conference on Sheet Metal, Apr 01.-04. 2007, Palermo, Sicily, Italy. Trans Tech Publication Ltd., Switzerland, pp 599-605

Taleb Araghi B, Manco GL, Bambach M, Hirt G (2009) Investigation into a new hybrid forming process: Incremental sheet forming combined with stretch forming. CIRP Annals-Manufacturing Technology 58(1):225-228

Vanderhasten M, Rabet L, Verlinden B (2008) Ti-6Al-4 V: deformation map and modelisation of tensile behaviour. Materials \& Design 29(6):1090-1098 